
human mobility through transportation

SUBJECT AREAS:

STATISTICAL PHYSICS

STATISTICS

Received

29 October 2014

Accepted

9 February 2015

Published

16 March 2015

\section{Correspondence and} requests for materials should be addressed to

K.Z. (kai.zhao@cs.

helsinki.fi) or S.T.

(sasu.tarkoma@cs.

helsinki.fi) \title{
modality decomposition
}

\author{
Kai Zhao ${ }^{1,5}$, Mirco Musolesi ${ }^{2}$, Pan $\mathrm{Hui}^{3}$, Weixiong $\mathrm{Rao}^{4} \&$ Sasu Tarkoma ${ }^{1,5}$
}

\begin{abstract}
'Department of Computer Science, University of Helsinki, Helsinki, Finland, '2School of Computer Science, University of Birmingham, Birmingham, UK, ${ }^{3}$ Department of Computer Science and Engineering, The Hong Kong University of Science and Technology, Hong Kong, ${ }^{4}$ School of Software Engineering, Tongji University, Shanghai, China, ${ }^{5}$ Helsinki Institute for Information Technology, HIIT, Helsinki, Finland.
\end{abstract}

Human mobility has been empirically observed to exhibit Lévy flight characteristics and behaviour with power-law distributed jump size. The fundamental mechanisms behind this behaviour has not yet been fully explained. In this paper, we propose to explain the Lévy walk behaviour observed in human mobility patterns by decomposing them into different classes according to the different transportation modes, such as Walk/Run, Bike, Train/Subway or Car/Taxi/Bus. Our analysis is based on two real-life GPS datasets containing approximately 10 and 20 million GPS samples with transportation mode information. We show that human mobility can be modelled as a mixture of different transportation modes, and that these single movement patterns can be approximated by a lognormal distribution rather than a power-law distribution. Then, we demonstrate that the mixture of the decomposed lognormal flight distributions associated with each modality is a power-law distribution, providing an explanation to the emergence of Lévy Walk patterns that characterize human mobility patterns.

nderstanding human mobility is crucial for epidemic control ${ }^{1-4}$, urban planning ${ }^{5,6}$, traffic forecasting systems ${ }^{7,8}$ and, more recently, various mobile and network applications ${ }^{9-13}$. Previous research has shown that trajectories in human mobility have statistically similar features as Lévy Walks by studying the traces of bank notes ${ }^{14}$, cell phone users' locations ${ }^{15}$ and GPS $^{16-19}$. According to the this model, human movement contains many short flights and some long flights, and these flights follow a power-law distribution.

Intuitively, these long flights and short flights reflect different transportation modalities. Figure 1 shows a person's one-day trip with three transportation modalities in Beijing based on the Geolife dataset (Table 1) 20-22 $^{2}$ Starting from the bottom right corner of the figure, the person takes a taxi and then walks to the destination in the top left part. After two hours, the person takes the subway to another location (bottom left) and spends five hours there. Then the journey continues and the person takes a taxi back to the original location (bottom right). The short flights are associated with walking and the second short-distance taxi trip, whereas the long flights are associated with the subway and the initial taxi trip. Based on this simple example, we observe that the flight distribution of each transportation mode is different.

In this paper, we study human mobility with two large GPS datasets, the Geolife and Nokia MDC datasets (approximately 20 million and 10 million GPS samples respectively), both containing transportation mode information such as Walk/Run, Bike, Train/Subway or Car/Taxi/Bus. The four transportation modes (Walk/ Run, Bike, Train/Subway and Car/Taxi/Bus) cover the most frequently used human mobility cases. First, we simplify the trajectories obtained from the datasets using a rectangular model, from which we obtain the flight length ${ }^{16}$. Here a flight is the longest straight-line trip from one point to another without change of direction ${ }^{16,19}$. One trail from an origin to a destination may include several different flights (Fig. 1). Then, we determine the flight length distributions for different transportation modes. We fit the flight distribution of each transportation mode according to the Akaike information criteria ${ }^{23}$ in order to find the best fit distribution.

We show that human movement exhibiting different transportation modalities is better fitted with the lognormal distribution rather than the power-law distribution. Finally, we demonstrate that the mixture of these transportation mode distributions is a power-law distribution based on two new findings: first, there is a significant positive correlation between consecutive flights in the same transportation mode, and second, the elapsed time in each transportation mode is exponentially distributed. 


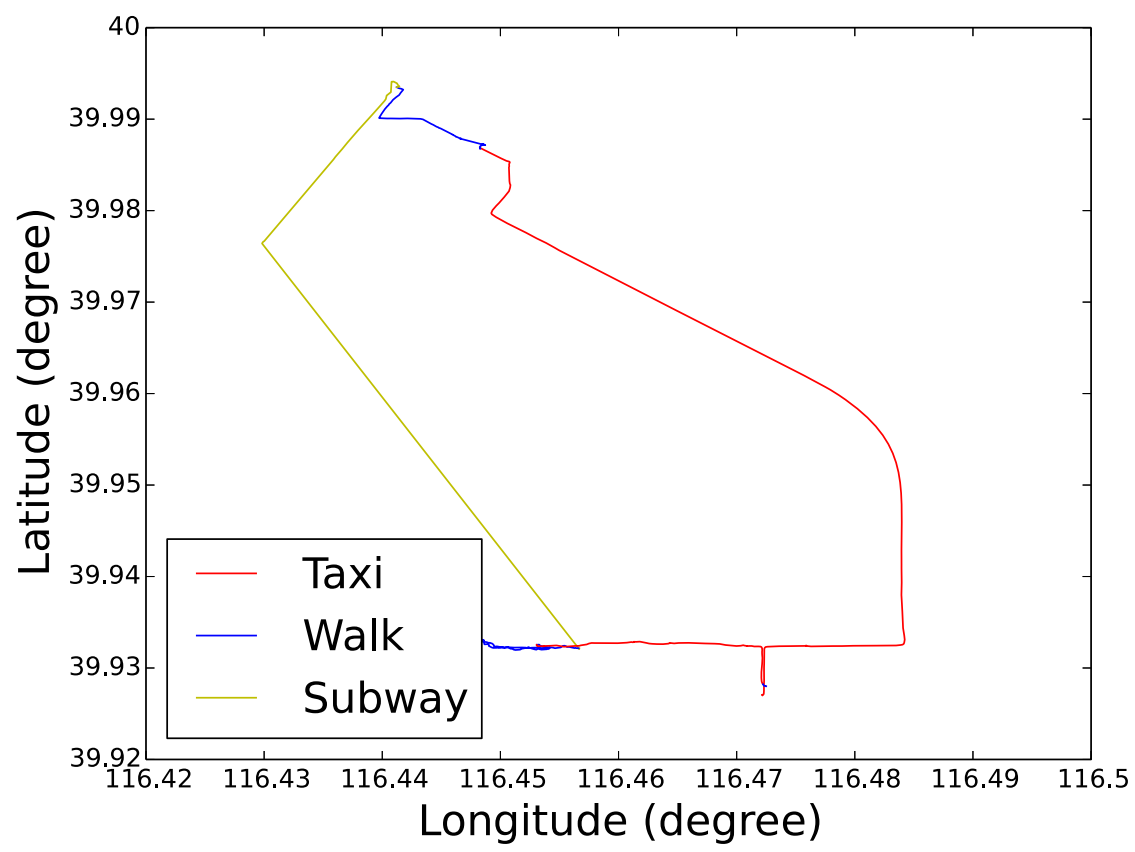

Figure 1 Illustration of a synthetic trail (taxi, walk, subway, walk, taxi, walk) for one day trip and their corresponding flights. This figure shows that the flight distribution of each transportation mode (walk, taxi, subway) is very different.

The contribution of this paper is twofold. First, we extract the distribution function of displacement with different transportation modes. This is important for many applications ${ }^{7-11}$. For example, a population-weighted opportunities (PWO) model $^{24}$ has been developed to predict human mobility patterns in cities. They find that there is a relatively high mobility at the city scale due to highly developed traffic systems inside cities. Our results significantly deepen the understanding of urban human mobilities with different transportation modes. Second, we demonstrate that the mixture of different transportations can be approximated as a truncated Lévy Walk. This result is a step towards explaining the emergence of Lévy Walk patterns in human mobility.

\section{Results}

Power-law fit for overall flight. First, we fit the flight length distribution of the Geolife and Nokia MDC datasets regardless of transportation modes (see Methods section). We fit truncated power-law, lognormal, power-law and exponential distribution (see Supplementary Table S1). We find that the overall flight length $(l)$ distributions fit a truncated power-law $P(l) \propto l^{\alpha} e^{\gamma l}$ with exponent $\alpha$ as 1.57 in the Geolife dataset $(\gamma=0.00025)$ and 1.39 in the Nokia MDC dataset $(\gamma=0.00016)$ (Fig. 2), better than other alternatives such as power-law, lognormal or exponential. Figure 2 illustrates the PDFs and their best fitted distributions according to

\begin{tabular}{|c|c|c|}
\hline & Geolife & Nokia MDC \\
\hline Location & Beijing & Geneva \\
\hline Measurement & GPS & GPS \\
\hline Number of samples & $24,876,978$ & $11,077,061$ \\
\hline Duration & 5 years & 1.5 year \\
\hline Accuracy & $3 \mathrm{~m}$ & $3 \mathrm{~m}$ \\
\hline Sampling interval & $1-5 s$ & $10 \mathrm{~s}$ \\
\hline Number of participants & 182 & 200 \\
\hline $\begin{array}{l}\text { Number of flights with } \\
\text { transportation mode }\end{array}$ & 202,702 & 224,723 \\
\hline
\end{tabular}

Akaike weights. The best fitted distribution (truncated power-law) is represented as a solid line and the rest are dotted lines. We use logarithm bins to remove tail noises ${ }^{16,25}$. Our result is consistent with previous research (Refs. 14-19), and the exponent $\alpha$ is close to their results.

We show the Akaike weights for all fitted distributions in the Supplementary Table S2. The Akaike weight is a value between 0 and 1. The larger it is, the better the distribution is fitted ${ }^{23,25}$. The Akaike weights of the power-law distributions regardless of transportation modes are 1.0000 in both datasets. The p-value is less than 0.01 in all our tests, which means that our results are very strong in terms of statistical significance. Note that here the differences between fitted distributions are not remarkable as shown in the Fig. 2, especially between the truncated power-law and the lognormal distribution. We use the loglikelihood ratio to further compare these two candidate distributions. The loglikelihood ratio is positive if the data is more likely in the power-law distribution, and negative if the data is more likely in the lognormal distribution. The loglikelihood ratio is 1279.98 and 3279.82 (with the significance value $p<0.01$ ) in the Geolife and the NokiaMDC datasets respectively, indicating that the data is much better fitted with the truncated power-law distribution.

Lognormal fit for single transportation mode. However, the distribution of flight lengths in each single transportation mode is not well fitted with the power-law distribution. Instead, they are better fitted with the lognormal distribution (see Supplementary Table S2). All the segments of each transportation flight length are best approximated by the lognormal distribution with different parameters. In Fig. 3 and Supplementary Fig. S1, we represent the flight length distributions of Walk/Run, Bike, Subway/Train and Car/ Taxi/Bus in the Geolife and the Nokia MDC dataset correspondingly. The best fitted distribution (lognormal) is represented as a solid line and the rest are dotted lines.

Table 2 shows the fitted parameter for all the distributions ( $\alpha$ in the truncated power-law, $\mu$ and $\sigma$ in the lognormal). We can easily find that the $\mu$ is increasing over these transportation modes (Walk/Run, Bike, Car/Taxi/Bus and Subway/Train), identifying an increasing average distance. Compared to Walk/Run, Bike or Car/Taxi/Bus, 


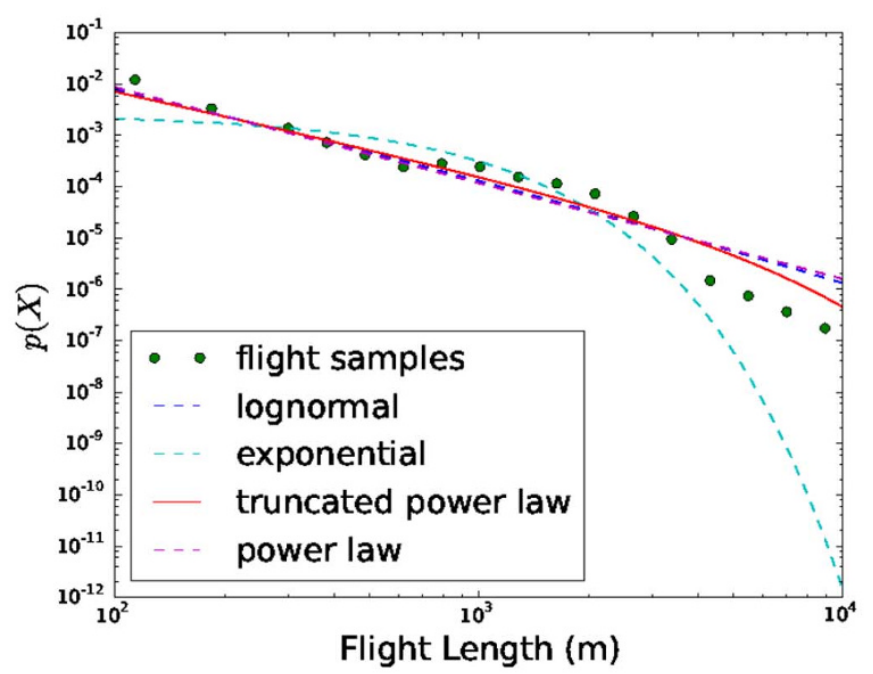

(a) Geolife

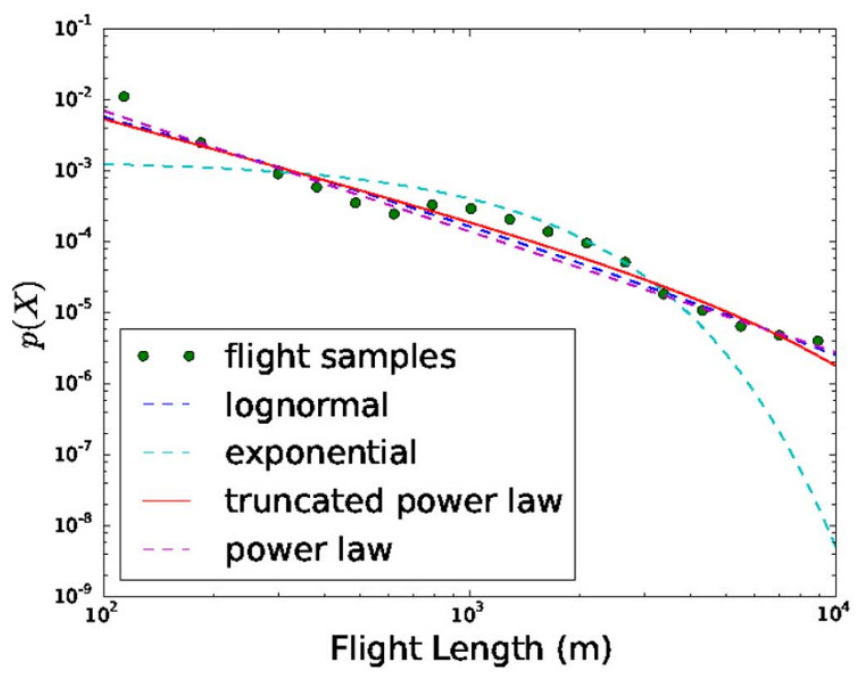

(b)Nokia MDC

Figure 2 Power-law fit for overall flight. (a-b) Power-law fitting of all flights regardless of transportation modes in the Geolife and the Nokia MDC dataset. The green points refer to the flight length samples obtained from the GeoLife and the Nokia MDC dataset, while the solid red line represents the best fitted distribution according to Akaike weights. The overall flight length distribution regardless of transportation modes is well fitted with a truncated power-law distribution.

the flight distribution in Subway/Train mode is more right-skewed, which means that people usually travel to a more distant location by Subway/Train.

It must be noted that our findings for the Car/Taxi/Bus mode are different from these recent research results ${ }^{26,27}$, which also investigated the case of a single transportation mode, and found that the scaling of human mobility is exponential by examining taxi GPS datasets. The differences are mainly because few people tend to travel a long distance by taxi due to economic considerations. So the displacements in their results decay faster than those measured in our Car/Taxi/Bus mode cases.

Mechanisms behind the power-law pattern. We characterize the mechanism of the power-law pattern with Lévy flights by mixing the lognormal distributions of the transportation modes. Previous research has shown that a mixture of lognormal distributions based on an exponential distribution is a power-law distribution ${ }^{30-33}$. Based on their findings, we demonstrate that the reason that human movement follows the Lévy Walk pattern is due to the mixture of the transportation modes they take.

We demonstrate that the mixture of the lognormal distributions of different transportation modes (Walk/Run, Bike, Train/Subway or Car/Taxi/Bus) is a power-law distribution given two new findings: first, we define the change rate as the relative change of length between two consecutive flights with the same transport mode. The change rate in the same transportation mode is small over time. Second, the elapsed time between different transportation modes is exponentially distributed.

Lognormal in the same transportation mode. Let us consider a generic flight $l_{t}$. The flight length at next interval of time $l_{t+1}$, given the change rate $c_{t+1}$, is

$$
l_{t+1}=l_{t}+c_{t+1} l_{t}
$$

It has been found that the change rate $c_{t}$ in the same transportation mode is small over time $e^{9,22}$. The change rate $c_{t}$ reflects the correlation between two consecutive displacements in one trip. To obtain the pattern of correlation between consecutive displace- ments in each transportation mode, we plot the flight length point $\left(l_{t}, l_{t+1}\right)$ from the GeoLife dataset (Fig. 4). Here $l_{t}$ represents the $t$ th flight length and $l_{t+1}$ represents the $t+1$-th flight length in a consecutive trajectory in one transportation mode ${ }^{34}$. Figure 4 shows the density of flight lengths correlation in Car/Taxi/Bus, Walk/Run, Subway/Train and Bike correspondingly. $\left(l_{t}, l_{t+1}\right)$ are posited near the diagonal line, which identifies a clear positive correlation. Similar results are also found in the Nokia MDC dataset (see Supplementary Fig. S2).

We use the Pearson correlation coefficient to quantify the strength of the correlation between two consecutive flights in one transportation mode ${ }^{35}$. The value of Pearson correlation coefficient $r$ is shown in the Supplementary Table S3. The $p$ value is less than 0.01 in all the cases, identifying very strong statistical significances. $r$ is positive in each transportation mode and ranges from 0.3640 to 0.6445 , which means that there is a significant positive correlation between consecutive flights in the same transportation mode, and the change rate $c_{t}$ in the same transportation mode between two time steps is small.

The difference $c_{t}$ in the same transportation mode between two time steps is small due to a small difference $l_{t+1}-l_{t}$ in consecutive flights. We sum all the contributions as follows:

$$
\begin{aligned}
\sum_{t=0}^{T} c_{t} & =\sum_{t=0}^{T} \frac{l_{t+1}-l_{t}}{l_{t}} \\
& \approx \int_{0}^{T} \frac{d l}{l}=\ln \frac{l_{T}}{l_{0}} .
\end{aligned}
$$

We plot the change rate samples $c_{t}$ of the Car/Taxi/Bus mode from the Geolife dataset as an example in Supplementary Fig. S3. We observe that the change rate $c_{t}$ fluctuates in an uncorrelated fashion from one time interval to the other in one transportation mode due to the unpredictable character of the change rate. The Pearson correlation coefficient accepts the findings at the 0.03-0.13 level with pvalue less than 0.05 (see Supplementary Table S4). By the Central Limit Theorem, the sum of the change rate $c_{t}$ is normally distributed with the mean $\mu T$ and the variance $\sigma^{2} T$, where $\mu$ and $\sigma^{2}$ are the mean and variance of the change rate $c_{t}$ and $T$ is the elapsed time. Then we 


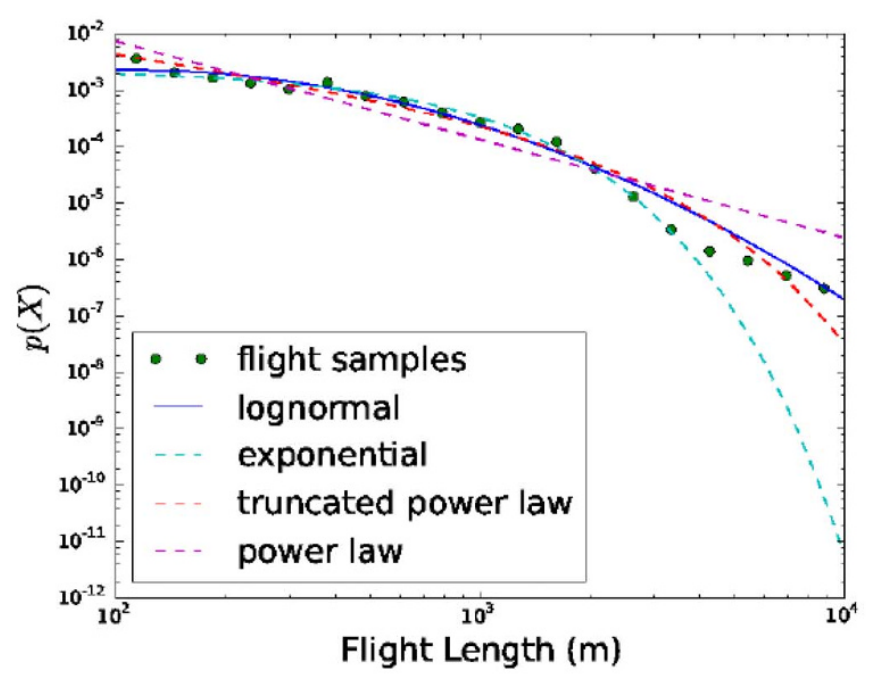

(a)Car/Taxi/Bus

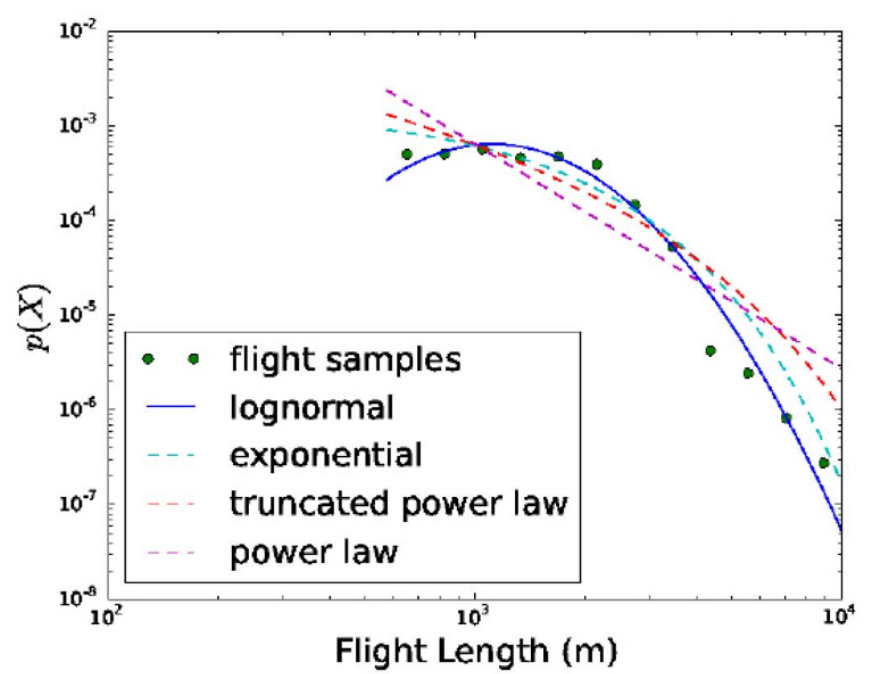

(c)Subway/Train

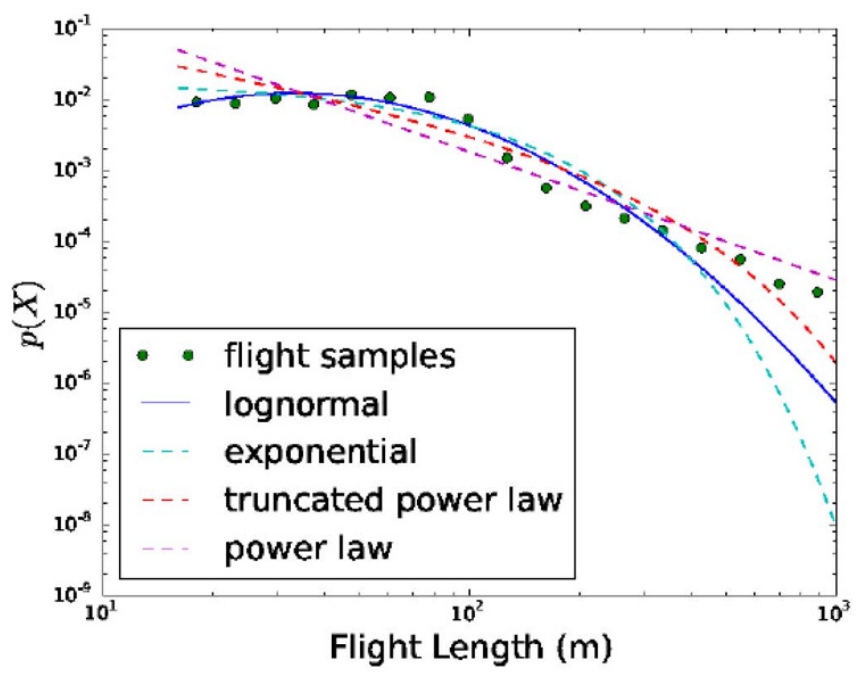

(b)Walk/Run

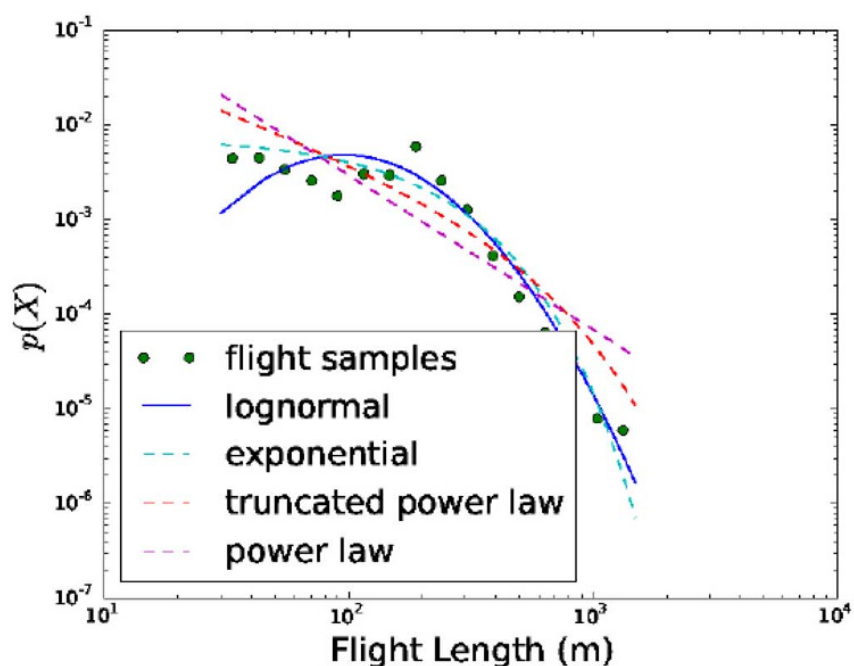

(d)Bike

Figure $3 \mid$ Lognormal fit for single transportation mode in the Geolife dataset. (a-d) Flight distribution of all transportation modes (Car/Taxi/Bus, Walk/Run, Subway/Train, Bike). The green points refer to the flight length samples obtained from the GeoLife, while the solid blue line represents the best fitted distribution according to Akaike weights. The flight length distribution in each transportation mode is well fitted with a lognormal distribution.

can assert that for every time step $t$, the logarithm of $l$ is also normally distributed with a mean $\mu t$ and variance $\sigma^{2} t^{36}$. Note here that $l_{T}$ is the length of the flight at the time $T$ after $T$ intervals of elapsed time. In the same transportation mode, the distribution of the flight length with the same change rate mean is lognormal, its density is given by

$$
P_{\text {singlemode }}(l)=\frac{1}{l \sqrt{2 \pi \sigma^{2} t}} \exp \left[-\frac{(\ln (l)-\mu t)^{2}}{2 \sigma^{2} t}\right],
$$

which corresponds to our findings that in each single transportation mode the flight length is lognormal distributed.

Table 2 | Parameters of fitted distributions in the Geolife and in the Nokia MDC datasets. The p-value is less than 0.01 in all the fitted distributions, identifying a strong statistical significance

\begin{tabular}{|c|c|c|c|c|}
\hline Dataset & Transportation Mode & Fitted Distribution & $p$ & Parameters \\
\hline \multirow[t]{4}{*}{ GeoLife } & Overall & Truncated Power-law & 0.00 & $\alpha=1.57, \gamma=0.00025$ \\
\hline & Walk/Run & Lognormal & 0.00 & $\mu=4.08, \sigma=0.76$ \\
\hline & Bike & Lognormal & 0.00 & $\mu=5.03, \sigma=0.68$ \\
\hline & Subway/Train & Lognormal & 0.00 & $\mu=7.27, \sigma=0.51$ \\
\hline \multirow{3}{*}{ Nokia MDC } & Overall & Truncated Power-law & 0.00 & $\alpha=1.39, \gamma=0.00016$ \\
\hline & Walk/Run & Lognormal & 0.00 & $\mu=4.58, \sigma=1.09$ \\
\hline & Subway/Train & Lognormal & 0.00 & $\mu=6.93, \sigma=0.94$ \\
\hline
\end{tabular}




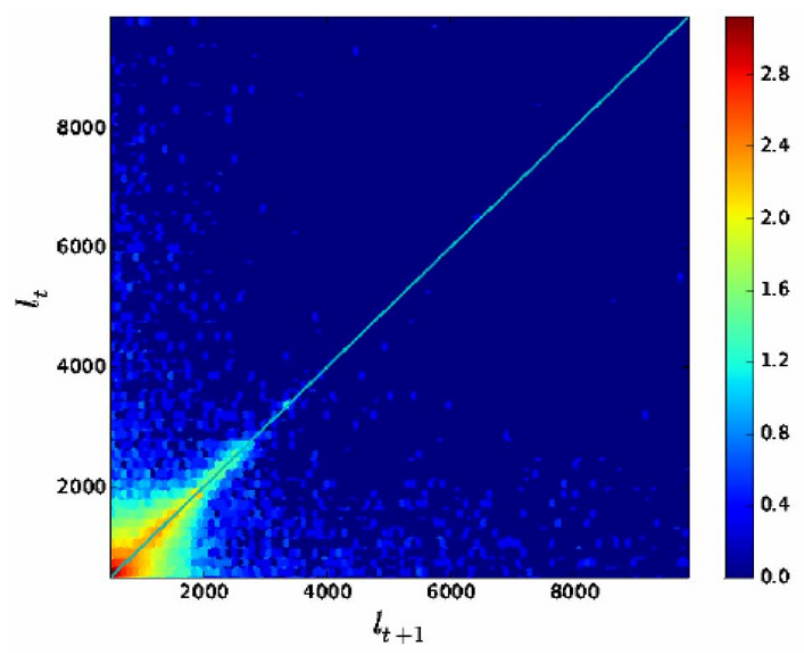

(a)Car/Taxi/Bus

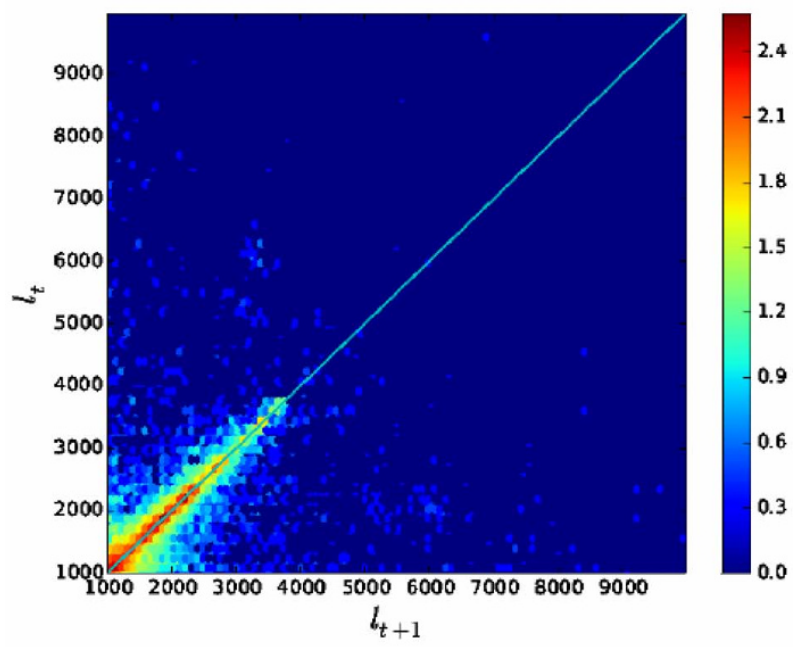

(c)Subway/Train

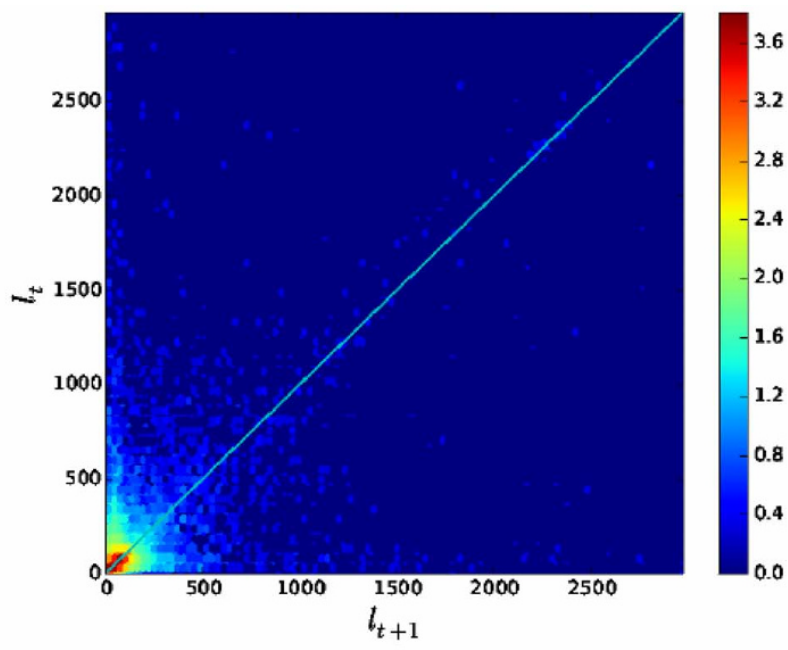

(b)Walk/Run

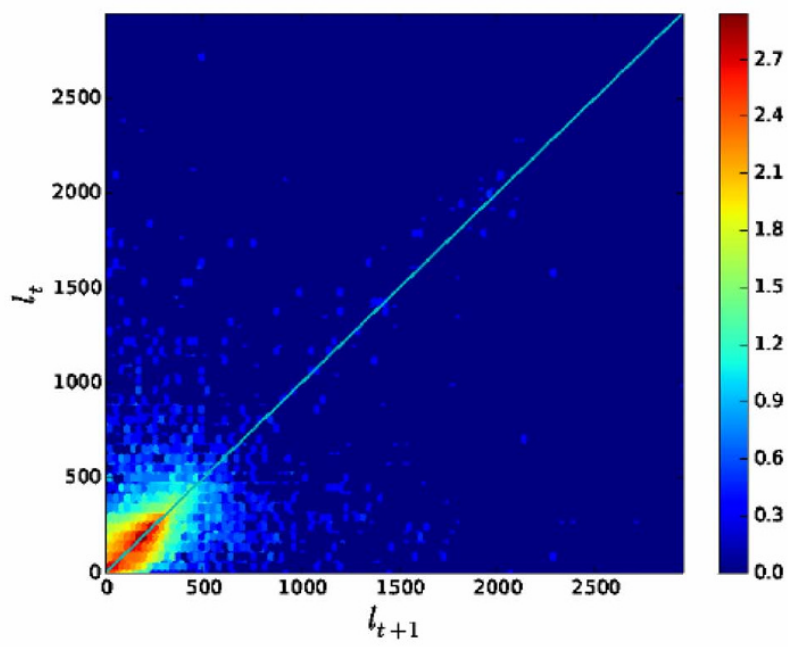

(d)Bike

Figure 4 Flight length correlation for each transportation mode. (a-d) Consecutive Flight length correlation of all transportation modes (Car/Taxi/ Bus, Walk/Run, Subway/Train, Bike) in the GeoLife dataset. A high density of points are near diagonal line $l_{t}=l_{t+1}$, identifying a small difference $l_{t+1}-l_{t}$ in the same transportation mode between two time steps.

Transportation mode elapsed time. We define elapsed time as the time spent in a particular transportation mode; we found that it is exponentially distributed. For example, the trajectory samples shown in Fig. 1 contain six trajectories with three different transportation modes, (taxi, walk, subway, walk, taxi, walk). Thus the elapsed time also consists of six samples $\left(t_{\text {taxi1 }}, t_{\text {walk } 1}, t_{\text {subway } 1}, t_{\text {walk } 2}, t_{\text {taxi } 2}, t_{\text {walk } 3}\right)$. The elapsed time $t$ is weighted exponentially between the different transportation modes (see Supplementary Fig. S4). Similar results are also reported in Refs. 26, 37. The exponentially weighted time interval is mainly due to a large portion of Walk/Run flight intervals. Walk/Run is usually a connecting mode between different transportation modes (e.g., the trajectory samples shown in Fig. 1), and Walk/Run usually takes much shorter time than any other modes. Thus the elapsed time decays exponentially. For example, $87.93 \%$ of the walk flight distance connecting other transportation modes is within 500 meters and the travelling time is within 5 minutes in the Geolife dataset.

Mixture of the transportation modes. Given these lognormal distributions $P_{\text {singlemode }}(l)$ in each transportation mode and the exponential elapsed time $t$ between different modes, we make use of mixtures of distributions. We obtain the overall human mobility probability by considering that the distribution of flight length is determined by the time $t$, the transportation mode change rate $c_{t}$ mean $\mu$ and variance $\sigma^{2}$. We obtain the distribution of single transportation mode distribution with the time $t$, the change rate mean $\mu$ and variance $\sigma^{2}$ fixed. We then compute the mixture over the distribution of $t$ since $t$ is exponentially distributed over different transportation modes with an exponential parameter $\lambda$. If the distribution of $l, p(l, t)$, depends on the parameter $t$. $t$ is also distributed according to its own distribution $r(t)$. Then the distribution of $l, p(l)$ is given by $p(l)=\int_{t=0}^{\infty} p(l, t) r(t) d t$. Here the $t$ in $p(l, t)$ is the same as the $t$ in the $r(t) . r(t)$ is the exponential distribution of elapsed time $t$ with an exponential parameter $\lambda$.

So the mixture (overall flight length $P_{\text {overall }}(l)$ ) of these lognormal distributions in one transportation mode given an exponential elapsed time (with an exponent $\lambda$ ) between each transportation mode is

$P_{\text {overall }}(l)=\int_{t=0}^{\infty} \lambda \exp (-\lambda t) \frac{1}{l \sqrt{2 \pi \sigma^{2} t}} \exp \left[-\frac{(\ln (l)-\mu t)^{2}}{2 \sigma^{2} t}\right] d t$ 
which can be calculated to give

$$
P_{\text {overall }}(l)=C l^{-\alpha^{\prime}},
$$

where the power law exponent $\alpha^{\prime}$ is determined by $\alpha^{\prime}=1-\frac{\mu}{\sigma^{2}}+{\frac{\sqrt{\mu^{2}+2 \lambda \sigma^{2}}}{\sigma^{2}}}^{31-33}$. The calculation to obtain $\alpha^{\prime}$ is given in Supplementary Note 1. If we substitute the parameters presented in Table 2, we will get the $\alpha^{\prime}=1.55$ in the Geolife dataset, which is close to the original parameter $\alpha=1.57$, and $\alpha^{\prime}=1.40$ in the Nokia MDC dataset, which is close to the original parameter $\alpha=1.39$. The result verifies that the mixture of these correlated lognormal distributed flights in one transportation mode given an exponential elapsed time between different modes is a truncated power-law distribution.

\section{Discussion}

Previous research suggests that it might be the underlying road network that governs the Lévy flight human mobility, by exploring the human mobility and examining taxi traces in one city in Sweden ${ }^{19}$. To verify their hypothesis, we use a road network dataset of Beijing containing 433,391 roads with 171,504 conjunctions and plot the road length distribution ${ }^{16,28,29}$. As shown in Supplementary Fig. S5, the road length distribution is very different to our power-law fit in flights distribution regardless of transportation modes. The $\alpha$ in road length distribution is 3.4, much larger than our previous findings $\alpha=$ 1.57 in the Geolife and $\alpha=1.39$ in the Nokia MDC. Thus the underlying street network cannot fully explain the Lévy flight in human mobility. This is mainly due to the fact that it does not consider many long flights caused by metro or train, and people do not always turn even if they arrive at a conjunction of a road. Thus the flight length tails in the human mobility should be much larger than those in the road networks.

\section{Methods}

Data Sets. We use two large real-life GPS trajectory datasets in our work, the Geolife datase $^{20}$ and the Nokia MDC dataset ${ }^{38}$. The key information provided by these two datasets is summarized in Table 1 . We extract the following information from the dataset: flight lengths and their corresponding transportation modes.

Geolife $^{20-22}$ is a public dataset with 182 users' GPS trajectory over five years (from April 2007 to August 2012) gathered mainly in Beijing, China. This dataset contains over 24 million GPS samples with a total distance of 1,292,951 kilometers and a total of 50,176 hours. It includes not only daily life routines such as going to work and back home in Beijing, but also some leisure and sports activities, such as sightseeing, and walking in other cities. The transportation mode information in this dataset is manually logged by the participants.

The Nokia MDC datase ${ }^{38}$ is a public dataset from Nokia Research Switzerland that aims to study smartphone user behaviour. The dataset contains extensive smartphone data of two hundred volunteers in the Lake Geneva region over one and a half years (from September 2009 to April 2011). This dataset contains 11 million data points and the corresponding transportation modes.

Obtaining Transportation Mode and The Corresponding Flight Length. We categorize human mobility into four different kinds of transportation modality: Walk/Run, Car/Bus/Taxi, Subway/Train and Bike. The four transportation modes cover the most frequently used human mobility cases. To the best of our knowledge, this article is the first work that examines the flight distribution with all kinds of transportation modes in both urban and intercity environments. In the Geolife dataset, users have labelled their trajectories with transportation modes, such as driving, taking a bus or a train, riding a bike and walking. There is a label file storing the transportation mode labels in each user's folder, from which we can obtain the ground truth transportation mode each user is taking and the corresponding timestamps. Similar to the Geolife dataset, there is also a file storing the transportation mode with an activity ID in the Nokia MDC dataset. We treat the transportation mode information in these two datasets as the ground truth.

In order to obtain the flight distribution in each transportation mode, we need to extract the flights. We define a flight as the longest straight-line trip from one point to another without change of direction ${ }^{16,19}$. One trail from an original to a destination may include several different flights (Fig. 1). In order to mitigate GPS errors, we recompute a position by averaging samples (latitude, longitude) every minute. Since people do not necessarily move in perfect straight lines, we need to allow some margin of error in defining the 'straight' line. We use a rectangular model to simplify the trajectory and obtain the flight length: when we draw a straight line between the first point and the last point, the sampled positions between these two endpoints are at a distance less than 10 meters from the line. The same trajectory simplification mechanism has been used in other articles which investigates the Lévy walk nature of human mobility ${ }^{16}$. We map the flight length with transportation modes according to timestamp in the Geolife dataset and activity ID in the Nokia MDC dataset and obtain the final (transportation mode, flight length) patterns. We obtain 202,702 and 224,723 flights with transportation mode knowledge in the Geolife and Nokia MDC dataset, respectively.

Identifying the Scale Range. To fit a heavy tailed distribution such as a power-law distribution, we need to determine what portion of the data to fit $\left(x_{\min }\right)$ and the scaling parameter $(\alpha)$. We use the methods from Refs. 30,39 to determine $x_{\min }$ and $\alpha$. We create a power-law fit starting from each value in the dataset. Then we select the one that results in the minimal Kolmogorov-Smirnov distance, between the data and the fit, as the optimal value of $x_{\text {min }}$. After that, the scaling parameter $\alpha$ in the powerlaw distribution is given by

$$
\alpha=1+n\left(\sum_{i=1}^{n} \ln \frac{x_{i}}{x_{\min }}\right)^{-1}
$$

where $x_{i}$ are the observed values of $x_{i}>x_{\min }$ and $n$ is number of samples.

Akaike weights. We use Akaike weights to choose the best fitted distribution. An Akaike weight is a normalized distribution selection criterion ${ }^{23}$. Its value is between 0 and 1 . The larger the value is, the better the distribution is fitted.

Akaike's information criterion (AIC) is used in combination with Maximum likelihood estimation (MLE). MLE finds an estimator of $\hat{\theta}$ that maximizes the likelihood function $L(\hat{\theta} \mid$ data $)$ of one distribution. AIC is used to describe the best fitting one among all fitted distributions,

$$
A I C=-2 \log (L(\hat{\theta} \mid \text { data }))+2 K .
$$

Here $\mathrm{K}$ is the number of estimable parameters in the approximating model.

After determining the AIC value of each fitted distribution, we normalize these values as follows. First of all, we extract the difference between different AIC values called $\Delta_{i}$

$$
\Delta_{i}=A I C_{i}-A I C_{\min } .
$$

Then Akaike weights $W_{i}$ are calculated as follows,

$$
W_{i}=\frac{\exp \left(-\Delta_{i} / 2\right)}{\sum_{r=1}^{R} \exp \left(-\Delta_{i} / 2\right)} .
$$

1. Ni, S. \& Weng, W. Impact of travel patterns on epidemic dynamics in heterogeneous spatial metapopulation networks. Physical Review E 79, 016111 (2009).

2. Balcan, D. \& Vespignani, A. Phase transitions in contagion processes mediated by recurrent mobility patterns. Nat Phys 7, 7 (2011).

3. Belik, V., Geisel, T. \& Brockmann, D. Natural human mobility patterns and spatial spread of infectious diseases. Phys. Rev. X 1, 011001 (2011)

4. Colizza, V., Barrat, A., Barthelemy, M., Valleron, A.-J. \& Vespignani, A. Modeling the worldwide spread of pandemic influenza: baseline case and containment interventions. PLoS Med 4, e13 (2007)

5. Zheng, Y., Liu, Y., Yuan, J. \& Xie, X. Urban computing with taxicabs. In Proceedings of the ACM International Joint Conference on Pervasive and Ubiquitous Computing (UbiComp), Beijing, China. ACM. (2011, September).

6. Yuan, J., Zheng, Y. \& Xie, X. Discovering regions of different functions in a city using human mobility and pois. In Proceedings of the 17th ACM SIGKDD Conference on Knowledge Discovery and Data Mining (KDD), San Diego, USA. ACM. (2012, August).

7. Jung, W.-S., Wang, F. \& Stanley, H. E. Gravity model in the korean highway. EPL (Europhysics Letters) 81, 48005 (2008)

8. Goh, S., Lee, K., Park, J. S. \& Choi, M. Y. Modification of the gravity model and application to the metropolitan seoul subway system. Phys. Rev. E 86, 026102 (2012).

9. Hemminki, S., Nurmi, P. \& Tarkoma, S. Accelerometer-based transportation mode detection on smartphones. In Proceedings of the 11th ACM Conference on Embedded Network Sensor Systems (SenSys), Roma, Italy. ACM. (2013, November).

10. Zheng, Y., Chen, Y., Li, Q., Xie, X. \& Ma, W.-Y. Understanding transportation modes based on gps data for web applications. TWEB 4 (2010).

11. Zheng, Y., Liu, L., Wang, L. \& Xie, X. Learning transportation mode from raw gps data for geographic applications on the web. In Proceedings of the 17th International World Wide Web Conference (WWW), Beijing, China. ACM. (2008, April).

12. Hui, P., Lindgren, A. \& Crowcroft, J. Empirical evaluation of hybrid opportunistic networks. In Proceedings of The 1st International Conference on COMunication Systems and NETworks(COMSNETS), Bangalore, India. IEEE. (2009, January). 
13. Rao, W., Zhao, K., Hui, P., Zhang, Y. \& Tarkoma, S. Towards maximizing timely content delivery in delay tolerant networks. IEEE Transactions on Mobile Computing 99, 1 (2014).

14. Brockmann, D., Hufnagel, L. \& Geisel, T. The scaling laws of human travel. Nature 439, 462-465 (2006).

15. Gonzalez, M. C., Hidalgo, C. A. \& Barabasi, A.-L. Understanding individual human mobility patterns. Nature 453, 779-782 (2008).

16. Rhee, I. et al. On the levy-walk nature of human mobility. IEEE/ACM Trans. Netw. 19, 630-643 (2011)

17. Yan, X.., Han, X.., Wang, B. \& Zhou, T. Diversity of individual mobility patterns and emergence of aggregated scaling laws. Sci. Rep. 3, 2678; DOI:10.1038/ srep02678 (2013).

18. Han, X.-P., Hao, Q., Wang, B.-H. \& Zhou, T. Origin of the scaling law in human mobility: hierarchy of traffic systems. Phys. Rev. E 83, 036117 (2011).

19. Jiang, B., Yin, J. \& Zhao, S. Characterizing the human mobility pattern in a large street network. Phys. Rev. E 80, 021136 (2009).

20. Zheng, Y., Xie, X. \& Ma, W.-Y. Geolife: a collaborative social networking service among user, location and trajectory. IEEE Data Eng. Bull. 33, 32-39 (2010).

21. Zheng, Y., Zhang, L., Xie, X. \& Ma, W.-Y. Mining interesting locations and travel sequences from gps trajectories. In Proceedings of the 18th International World Wide Web Conference (WWW), Madrid, Spain. ACM. (2009, April).

22. Zheng, Y., Li, Q., Chen, Y., Xie, X. \& Ma, W.-Y. Understanding mobility based on gps data. In Proceedings of the ACM International Joint Conference on Pervasive and Ubiquitous Computing (UbiComp), Orlando, USA. ACM. (2008, September).

23. Burnham, K. \& Anderson, D. Model Selection and Multi-Model Inference: A Practical Information-Theoretic Approach. (Springer, 2010).

24. Yan, X-Y., Zhao, C., Fan, Y., Di, Z. \& Wang, W.-X. Universal predictability of mobility patterns in cities. J. R. Soc. Interface 11, 20140834; DOI:10.1098/ rsif.2014.0834 (2014)

25. Alstott, J., Bullmore, E. \& Plenz, D. Powerlaw: a python package for analysis of heavy-tailed distributions. PLoS ONE 9, e85777; DOI:10.1371/journal.pone. 0085777 (2014).

26. Liang, X., Zhao, J., Dong, L. \& Xu, K. Unraveling the origin of exponential law in intra-urban human mobility. Sci. Rep. 3, 2983; DOI:10.1038/srep02983 (2013).

27. Liang, X., Zheng, X., Lv, W., Zhu, T. \& Xu, K. The scaling of human mobility by taxis is exponential. Physica A 391, 2135-2144 (2012).

28. Yuan, N. J. \& Zheng, Y. Segmentation of urban areas using road networks. Technical Report (2012). Available at: http://research.microsoft.com/pubs/ 168518/mapsegmentation.pdf. (Accessed: 22nd January 2015).

29. Song, R., Sun, W., Zheng, B. \& Zheng, Y. Press: A novel framework of trajectory compression in road networks. In Proceedings of The 40th International Conference on Very Large Data Bases (VLDB), Hangzhou, China. IEEE. (2014, September).

30. Newman, M. E. J. Power laws, pareto distributions and zipf's law. Contemporary Physics 46, 323 (2005)

31. Huberman, B. A. \& Adamic, L. A. The nature of markets in the world wide web. Quarterly Journal of Economic Commerce. DOI:10.2139/ssrn.166108 (2000).

32. Huberman, B. A. \& Adamic, L. A. Evolutionary dynamics of the world wide web. arXiv:cond-mat/9901071 (1999).

33. Mitzenmacher, M. A brief history of generative models for power law and lognormal distributions. Internet Mathematics 1, 226-251 (2004).
34. Wang, X.-W., Han, X.-P. \& Wang, B.-H. Correlations and scaling laws in human mobility. PLoS ONE 9, e84954; DOI:10.1371/journal.pone.0084954 (2014).

35. Cohen, J. Statistical Power Analysis for the Behavioral Sciences. 2nd edn (Routledge, 1988).

36. Ross, S. M. Stochastic Processes. 2nd edn (Wiley, New York, 1995).

37. Zhu, H. et al. Recognizing exponential inter-contact time in vanets. In Proceedings of The 29th IEEE Conference on Computer Communications (INFOCOM), Berlin, Germany. IEEE. (2010, March).

38. Kiukkonen, N., Blom, J., Dousse, O., Gatica-Perez, D. \& Laurila, J. Towards rich mobile phone datasets: Lausanne data collection campaign. In Proceedings of International Conference on Pervasive Services (ICPS), Berlin, Germany. ACM. (2010, July).

39. Clauset, A., Shalizi, C. \& Newman, M. Power-law distributions in empirical data. SIAM Review 51, 661-703 (2009).

\section{Acknowledgments}

The research at the University of Helsinki was supported by the DIGILE Internet of Things research program, ESENS project funded by Tekes and EIT ICT Labs. Mirco Musolesi would like to acknowledge the support of EPSRC through the grants "Trajectories of Depression" (P/L006340/1) and "The Uncertainty of Identity: Linking Spatiotemporal Information Between Virtual and Real Worlds" (EP/J005266/1). Weixiong Rao's work is partially supported by Shanghai Science and Technology Commission (15ZR1443000) and IBM Faculty award 2014. We thank Petteri Nurmi for discussions and feedback on the manuscript.

\section{Author contributions}

K.Z., M.M., P.H., W.R. and S.T. designed the research based on the initial idea by K.Z. and S.T.. K.Z. executed the experiments guided by M.M., P.H., W.R. and S.T.. K.Z. and S.T. performed statistical analyses, and prepared the figures. K.Z., M.M., P.H., W.R. and S.T. wrote the manuscript. All authors reviewed the manuscript.

\section{Additional information}

Supplementary information accompanies this paper at http://www.nature.com/ scientificreports

Competing financial interests: The authors declare no competing financial interests. How to cite this article: Zhao, K., Musolesi, M., Hui, P., Rao, W. \& Tarkoma, S. Explaining the power-law distribution of human mobility through transportation modality decomposition. Sci. Rep. 5, 9136; DOI:10.1038/srep09136 (2015).

This work is licensed under a Creative Commons Attribution 4.0 International License. The images or other third party material in this article are included in the article's Creative Commons license, unless indicated otherwise in the credit line; if the material is not included under the Creative Commons license, users will need to obtain permission from the license holder in order to reproduce the material. To view a copy of this license, visit http://creativecommons.org/licenses/by/4.0/ 